\section{Response to ten Broeke et al.}

We appreciate the thoughtful feedback by ten Broeke et al. ${ }^{1}$ on our manuscript entitled "MSH6 and PMS2 Germ-line Pathogenic Variants Implicated in Lynch Syndrome Are Associated with Breast Cancer." While we agree with most of the limitations they outline, which had already been included in our discussion, a few points warrant further response. First, we agree with the authors about the advantages of segregation analyses. However, we did not have sufficiently detailed family data to allow us to conduct these analyses. While standardized incidence ratio (SIR) analysis has limitations particularly when the external comparison group may not be comparable to the internal group, we were able to evaluate the specificity of our findings across four cancer types (breast, colon, endometrial, and ovarian), and only breast cancer had findings specific to MSH6 and PMS2. We would also like to emphasize that the magnitude of the SIR we found, using four times the number of cases in the study by ten Broeke, also found a similar SIR estimate for PMS2, namely 2.92 (95\% confidence interval $[\mathrm{CI}]=2.17-3.92$ ) for our study versus 3.8 $(95 \% \mathrm{CI}=1.9-6.8)$ for that of ten Broeke. The tighter confidence interval of our estimate is based upon the larger number of breast cancer cases in our study. Although we observed comparable SIRs for PMS2 with ten Broeke as well as comparable aggregate SIRs with that of Engle, the actual SIR estimates depend on the underlying age structure and the weights based on the prevalence of pathogenic variants per gene. The study by Engel included proportionately more individuals with pathogenic variants in $M L H 1$ and $M S H 2$, but the underlying age structure was not described. The study by Engle also did not include PMS2. Our aggregate SIR estimates may be lower because a higher incidence was used to estimate the expected number of breast cancer cases as well as a younger age cohort. Our cumulative risks are much higher, which is in line with what would be expected based on the high proportion of pathogenic variants in MSH6 and PMS2 in our study.

Ten Broeke et al. ${ }^{1}$ hypothesize that the relatively high frequency of MSH6 and PMS2 pathogenic variants in the general population could have inflated the breast cancer SIRs calculated for these two genes in our study. Our ascertainment method and the frequency of pathogenic variants in these genes may have influenced the calculated SIRs; however, this is unlikely to be the sole explanation for the observed increased risk of breast cancer. The higher frequency of MSH6 and PMS2 pathogenic variants in the general population compared to $M L H 1$ and $M S H 2$ has been suggested by not only Win et al. ${ }^{2}$ but also by Espenchied et al. ${ }^{3}$ in addition to our results. ${ }^{1}$ By comparing the cancer distribution across our cohort, we were able to compare the magnitude of the SIRs across cancer types, which had similar methods of ascertainment. The cancer distribution is different between genes, with increased breast cancer only associated with MSH6 and PMS2 suggesting that the increased SIRs are not simply due to the frequency of pathogenic variants in MSH6 and PMS2.

Furthermore, other groups have also reported an association between MSH6 and PMS2 and breast cancer. Espenschied et $\mathrm{al}^{3}$ found that the frequency of PMS2 pathogenic variants in breast cancer-only patients was significantly higher than published population frequencies $(p=0.0006)$, and Couch et al. ${ }^{4}$ reported an association with breast cancer for MSH6 using data from the Exome Aggregation Consortium (ExAC) as a reference control (odds ratio $[\mathrm{OR}]=1.93 ; 95 \% \mathrm{CI}$, $1.16-3.27, p=0.01)\left(\right.$ ref. $\left.^{2}\right)$.

The efforts of the Prospective Lynch Syndrome Database are commendable, but we suspect that the breast cancer cumulative risks available on that website may not be representative of all women with Lynch syndrome. This cohort is also likely subject to ascertainment bias, as study participants are recruited based on patients' perceived increased colorectal cancer risk and established clinical diagnostic criteria. ${ }^{5}$ In addition to our assessment of established Lynch syndrome clinical and testing criteria, Espenschied et al. also found that MSH6 and PMS2 pathogenic variant carriers are more likely to present with a hereditary breast and ovarian cancer phenotype, which may explain why pathogenic variants in these two genes have been historically underreported in the literature. While prospective studies are needed, new cancer associations are not likely to be delineated as long as clinical and research testing for Lynch syndrome continues to be based on previously established criteria (i.e., Amsterdam II criteria and Bethesda Guidelines), which are heavily based on colon cancer diagnoses.

Again, as stated in our manuscript, we recognize that this study has inherent limitations and largely agree with the points made by ten Broeke et al. We agree that our results need to be replicated before any standard breast cancer management recommendations are made. Ultimately, studies of population-based cohorts must be performed to minimize the ascertainment biases inherent in all of the studies to date.

However, we believe that our data on multigene panel testing adds to the body of knowledge regarding the phenotypic spectrum of Lynch syndrome, which appears to be broader than was previously appreciated.

\section{DISCLOSURE}

Maegan Roberts is an employee of GeneDx/BioReference Laboratories, Inc. and has a salary as the only disclosure. Wendy Chung is a former employee of BioReference Laboratories. 
Benjamin Solomon has multiple disclosures which include stock and employment with GeneDx/BioReference Laboratories. The other authors declare that they have no conflicts of interest.

Maegan E. Roberts, $M S^{1}$, Nur Zeinomar, PhD, $M P H^{2}$, Benjamin D. Solomon, $M D^{1}$, Mary Beth Terry, $P h D^{2}$ and Wendy K. Chung, $M D, P h D^{3}$

${ }^{1}$ GeneDx, Gaithersburg, Maryland, USA; ${ }^{2}$ Department of Epidemiology, Columbia University, New York, New York, USA; ${ }^{3}$ Department of Pediatrics and Medicine, Columbia University, New York, New York, USA. Correspondence:

Maegan E. Roberts (mroberts@genedx.com)

\section{REFERENCES}

1. ten Broeke SW, Brohet RM, Tops CM, et al. Lynch syndrome caused by germline PMS2 mutations: delineating the cancer risk. J Clin Oncol. Q4 2015;33:319-25.
2. Win AK, Jenkins MA, Dowty JG, et al. Prevalence and penetrance of major genes and polygenes for colorectal cancer. Cancer Epidemiol Biomarkers Prev. 2016;26:404-12.

3. Espenschied CR, La Duca H, Li S, et al. Multigene panel testing provides a new perspective on Lynch syndrome. J Clin Oncol. 2017;35:2568-75.

4. Couch FJ, Shimelis $\mathrm{H}, \mathrm{Hu} \mathrm{C}$, et al. Associations between cancer predisposition testing panel genes and breast cancer. JAMA Oncol. 2017;3:1190-96.

5. Moller P, Seppälä T, Bernstein I, et al. Cancer incidence and survival in Lynch syndrome patients receiving colonoscopic and gynaecological surveillance: first report from the prospective Lynch syndrome database. Gut. 2017:66:464-72.

Advance online publication 24 May 2018. doi:10.1038/s41436-018-0031-7 